\title{
Towards a multi-antigen multi-stage malaria vaccine
}

\author{
Adrian VS Hill, Sumi Biswas, Simon Draper, Thomas Rampling, Arturo Reyes-Sandoval \\ From Challanges in malaria research: Core science and innovation \\ Oxford, UK. 22-24 September 2014
}

A highly effective malaria vaccine is a major goal of glo-
bal health research and will likely require a multi-stage
product. Oxford researchers are developing the concept
of a highly effective multi-stage P. falciparum vaccine to
the point of proof-of-concept phase II testing in Europe,
prior to trials in malaria-endemic areas.

Remarkable recent advances in vaccine design for all four stages of the P. falciparum parasite's life-cycle allow testing of a multi-stage multi-component vaccine for the first time, with strong chances of success. These advances are i) the availability of a new vectored primeboost vaccination regime based on the chimpanzee adenovirus technology that has been found to induce exceptionally potent $\mathrm{CD} 8^{+} \mathrm{T}$ cell responses and high titre antibodies against multiple malaria antigens; ii) the development of an improved virus-like particle (VLP) version of the leading partially protective RTS,S sporozoite vaccine candidate, termed $\mathrm{R} 21$, that lacks the excess of HBsAg in RTS,S; iii) the identification, using a vector technology screen, of the blood-stage antigen $\mathrm{RH} 5$ as the first antigen to induce potent strain-transcending neutralization of blood-stage parasites in in vitro growth inhibition assays; and iv) the demonstration that antibodies against a mosquito-stage antigens that induce $100 \%$ transmission blocking against field isolates of P. falciparum in Africa are inducible by a new nanoparticle vaccine candidate.

In parallel similar approaches using vectors and VLPs are underway to target the pre-erythrocytic stages of $P$. vivax, including the hypnozoite, and a phase I trial of the vivax blood-stage vaccine candidate, PvRII, is nearing completion.

We are aiming to undertake phase I/II clinical trials to assess the $P$. falciparum pre-erythrocytic, blood-stage and mosquito-stage components individually, and then

University of Oxford, UK and take full advantage of:

- Convenient online submission

- Thorough peer review

- No space constraints or color figure charges

- Immediate publication on acceptance

- Inclusion in PubMed, CAS, Scopus and Google Scholar

- Research which is freely available for redistribution

Submit your manuscript at

C BioMed Central 